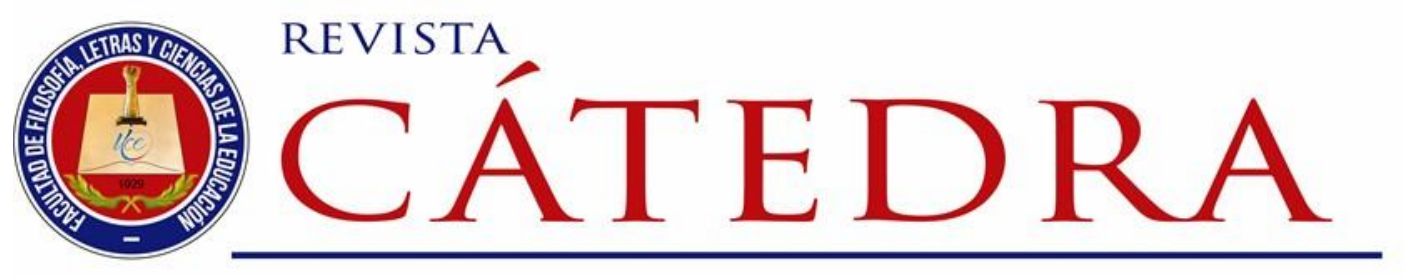

\title{
Innovación educativa y su incidencia en el profesorado
}

\section{Educational innovation and its impact on teachers}

\author{
Ruth Páez-Granja \\ Universidad Central del Ecuador, Quito, Ecuador \\ repaez@uce.edu.ec \\ https://orcid.org/0000-0002-7169-6821
}

\author{
Ana Beatriz Martínez-González \\ Universidad Central de Venezuela, Carcas, Venezuela \\ ana.b.martinez@ucv.ve \\ https://orcid.org/0000-0001-7301-251
}

(Recibido: 8/08/2019 Aceptado: 19/08/2019; Versión final recibida: 15/09/2019)

Cita del artículo: Páez-Granja, R. y Martínez-González, AB. (2019). Innovación educativa y su incidencia en el profesorado. Revista Cátedra, 2(3), 83-103.

\section{Resumen}

El presente artículo tiene como objetivo el análisis de los procesos de cambio e innovación en la educación y su incidencia en el profesorado. El tema adquiere particular relevancia debido a que la educación superior en Ecuador está pasando por importantes procesos de transformación que ameritan revisar los contenidos curriculares y la metodología de enseñanza, aspectos fundamentales que subyacen en todo proceso de innovación o cambio educativo y la adaptación del profesorado a estos. La metodología del presente trabajo consistió en el análisis de los principales postulados de Fullan (2002-2012), Havelock y Huberman (1980), Rutherford y Hall (1990), Rogers (2003), Marcelo (1995-2010), entre otros, que se han dedicado al estudio de los procesos de cambio en el contexto educativo y su impacto en el profesorado. Como resultado de este estudio se identifica como factor fundamental la participación proactiva de los docentes en la construcción y fortalecimiento de los procesos de innovación para la formación integral de los ciudadanos en un marco de compromiso social y ético con la educación en todos sus niveles. El trabajo concluye con un cuerpo de recomendaciones que supone, entre otras, la necesidad de involucrar al profesorado desde el inicio en los procesos de transformación educativa a través de programas de sensibilización, formación y acompañamiento.

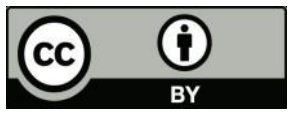




\section{Palabras clave}

Cambio educativo, innovación educativa, profesorado, educación superior.

\section{Abstract}

The purpose of this article is to analyze the processes of change and innovation in education and its impact on professors. The topic is relevant since higher education in Ecuador is going through an important transformation process that requires reviewing the curriculum content and the teaching methodology, key aspects that underlie any process of innovation or educational change and the adaptation of professors to these processes. The methodology consisted in the analysis of the main postulates of Fullan (2002-2012), Havelock and Huberman (1980), Rutherford and Hall (1990), Rogers (2003), Marcelo (1995-2010), among others, devoted to the impact of the processes of change in professors. As a result of this study, the proactive participation of teachers in the construction and strengthening of innovation processes is identified for the integral formation of citizens with a social and ethical commitment to the education at all levels. The work concludes with recommendations that implies the need to involve teachers from the beginning in the processes of educational transformation through awareness-raising, training and accompaniment programs.

\section{Keywords}

Educational change, educational innovation, professors, higher education.

\section{Introducción}

La educación superior en América Latina y en el mundo está condicionada tanto por la aceleración y transformación sistemática que atraviesa la sociedad como por las demandas que plantea la denominada sociedad del conocimiento. En tal sentido, la educación superior, a pesar de haber mostrado diversos tipos de desarrollo en las últimas décadas del siglo XX, al inicio del nuevo siglo se encuentra en crisis a nivel mundial, obligada a redefinirse permanentemente para enfrentar los nuevos desafíos. La masificación, la demanda de nuevas habilidades, la evolución de la tecnología y la creación acelerada y continua de conocimiento, exigen cambios constantes en el sistema educativo.

Referirse a los cambios en educación es reconocer las demandas que existen actualmente y que deben ser atendidas con prioridad por la sociedad en su conjunto, pues de ello depende la posibilidad de que surjan procesos de innovación que se espera contribuyan a elevar la calidad educativa. En este sentido, en un contexto de permanente transformación caracterizado por la mundialización, la masificación, la tecnologización y la multialfabetización entre otras tendencias; la apertura y adaptación al proceso de cambio permanente debe formar parte de la dinámica de las instituciones. Ello supone desarrollar estrategias que den respuesta a esas tendencias que pasan entre otros cambios, por la flexibilización del currículo.

No es casual que los desfases entre las demandas sociales y la respuesta de las instituciones educativas han sido motivo de múltiples reuniones, convenciones, congresos, a nivel mundial y local, con el fin de profundizar en el análisis de las nuevas realidades y establecer acuerdos y reformas, que permitan delinear propuestas, proyectos, estrategias, cuyo objetivo principal consiste en elevar la calidad de la educación. 
En el Ecuador se han desarrollado procesos de evaluación cuyos resultados han evidenciado serios cuestionamientos sobre la pertinencia y calidad de la educación superior. Al respecto, parte del balance se evidencia en lo siguiente:

Cabe resaltar como principales tensiones o problemas de la educación superior la cuestionable gestión en lo referente a: la calidad y pertinencia, la no aplicación del principio de igualdad de oportunidades e integralidad del sistema educativo, la calidad de la educación a distancia o virtual, la autonomía universitaria con responsabilidad social, la integralidad académica, la formación y la investigación (Larrea y Granados, 2016, pp. 155-235).

Es indudable que la sociedad ecuatoriana demanda cambios en la educación superior; pues ha sido y es una necesidad cumplir con lo más sustancial de la Declaración de Bolonia para la educación superior (1999), con el fin de estar acorde con los retos que plantea la sociedad del conocimiento. Se destacan en la Declaración, los aspectos referidos a la calidad, movilidad, diversidad y competitividad para procurar la adaptación de la oferta educativa a las demandas sociales. Estas demandas suponen cambios que requieren ser debatidos, analizados en espacios amplios de reflexión con los actores sociales, con los integrantes de la academia, con la comunidad universitaria. Ello, en efecto, contribuye a establecer procesos de elaboración de los nuevos modelos educativos, pedagógicos y construir los nuevos diseños curriculares de las carreras que cumplan el objetivo de formar a los futuros profesionales acorde con los nuevos escenarios sociales, que se relacionen con los modelos de sociedad y con los proyectos de desarrollo del país. Tal como lo han señalado Marcelo, Mayor y Gallego (2010):

Los cambios que se están produciendo en nuestra sociedad afectan a la educación y al trabajo docente. Una sociedad del conocimiento requiere de centros educativos orientados hacia la innovación y la calidad. Pero la innovación no es un mandato. No se puede ordenar (p.112).

En todo proceso de cambio se debe considerar la participación de aquellos que van a ser afectados por dicho cambio. Si se adelanta un proceso de transformación, el éxito del mismo parte de que los actores del proceso formen parte de su concepción e implementación. De acuerdo con Fullan (2002a), "para conseguir ciertas clases de propósitos, en este caso, los importantes objetivos educativos no se pueden imponer por mandato, porque lo que realmente importa para los complejos objetivos del cambio son las aptitudes, el pensamiento creativo y la acción comprometida" (p. 36). Todo proceso de cambio, de innovación, especialmente en educación, debe surgir de un contexto en el cual se identifiquen los problemas y se establezcan con los actores educativos las ideas, propuestas, proyectos, reformas, mandatos, modelos. Propuestas impuestas no surten el efecto deseado.

En este contexto argumentativo, el objetivo del presente trabajo consiste en analizar los principios teóricos que subyacen a los procesos de cambio e innovación en educación y su incidencia en el profesorado. Es necesario reflexionar sobre el hecho de que todo proceso de cambio e innovación educativa tal como se ha planteado en el caso de Ecuador (Breilth, 2017). y específicamente al considerar la Universidad Central de Ecuador (Páez, 2018). Está supeditado a un sinnúmero de factores tales como la capacitación del docente, la comprensión del nuevo modelo curricular, la capacidad de asumir y realizar los cambios que implican las reformas, y sobre todo, los niveles de afectación que se considera tendrían los docentes sobre su desempeño, entre otros. En definitiva, si no existe conocimiento, claridad y capacitación relacionados con los procesos que se van a emprender, se podrían generar dificultades en su implementación. Se trata de encauzar los lineamientos 
sociológicos, psicológicos, pedagógicos del cambio y la innovación educativa para priorizar procesos inclusivos y/o construir nuevos, que fortalezcan los desarrollados por cada sociedad considerando al docente como un actor, facilitador de saberes con lineamientos críticos y constructivistas, relacionándolos con el contexto familiar y el entorno sociocultural.

Este trabajo está dividido en cuatro partes. En el primero se aborda el tema del cambio educativo, luego se expone una visión panorámica acerca de los procesos de innovación y cambio educativo y finalmente se discuten los aspectos y los factores que intervienen en todo proceso de innovación educativa.

\section{Acerca del cambio educativo}

El cambio educativo no es algo nuevo. Desde hace muchas décadas a nivel mundial se viene discutiendo este tema. La naturaleza del cambio, debido a los avances en las diferentes disciplinas y al impacto de las tecnologías en la sociedad, es múltiple y profunda. Afecta los sistemas de producción, las relaciones de poder, la política, la cultura, y por supuesto la educación produciendo intensas transformaciones. En el caso educativo, pasamos del libro a la red, al aprendizaje móvil y abierto y permanente.

La cultura del siglo XXI demanda diversos tipos de alfabetización: la alfabetización audiovisual, digital e informacional. El paradigma educativo se mueve para incorporar la educación a distancia, el aprendizaje mixto y aprendizaje colaborativo. Los medios están cambiando la manera como interactuamos, presentamos las ideas y la información y nos comunicamos. La demanda de formación supone atender aprendizajes nuevos y competencias en ambientes de aprendizajes virtuales y personalizados, acompañando el desarrollo de la formación a lo largo de la vida.

En México, por ejemplo, tal como señala Frida Díaz-Barriga, docente de la UNAM, en su artículo Reformas Curriculares y Cambio Sistémico (2012) que, desde la década de los 90, se han desarrollado procesos de innovación curricular para la transformación educativa, con una serie de modelos, cambios, con gran potencial educativo, que se orientan a alcanzar la tan mentada calidad educativa (pp. 23-40). No obstante, sigue proliferando en los procesos de reforma, un enfoque centralizado y de implantación de arriba hacia abajo sin que se logre articular una dinámica de cambio sistémico. Uno de los problemas que destaca la autora es la carencia o insuficiente información con relación a los procesos y condiciones de aplicación de los cambios, lo que impide su desarrollo exitoso en la diversidad de contextos en donde se ha pretendido su implementación. Esto ha generado que los actores educativos no quieran involucrarse en los procesos y si les corresponde, lo hacen por obligación, mas no con el convencimiento de que estos cambios permitirán transformar las creencias y prácticas educativas para atender las demandas de la sociedad.

Los cambios generalmente vienen de arriba hacia abajo y de afuera hacia adentro, con el agravante de que el profesorado lo considera como algo impuesto. Al respecto, Murrillo y Krichesky (2012) sostienen que, "el cambio no es lineal puesto que lo que sucede es que cada fase puede tener efectos retroactivos o alterar las decisiones tomadas en una etapa anterior" (p. 4). Estos procesos de cambio, que pueden ser parte de reformas educativas, se vienen realizando a nivel mundial y responden a la creciente y globalizada sociedad del conocimiento, a la incorporación de las TIC, a modelos internacionales, a enfoques económicos-empresariales; propuestas que se encuentran orientadas a la evaluación de la calidad ligada al financiamiento de la educación, la certificación y la acreditación. Ello se acompaña de la dificultad de que estos estándares de calidad son unificados y generales y no se toma en cuenta la diversidad social, cultural e institucional de cada comunidad.

Licencia Creative Commons Atribución 4.0 Internacional (CC BY 4.0)

Revista Cátedra, 2(3), pp. 83-103, septiembre-diciembre 2019. e-ISSN: 2631-2875

https://doi.org/10.29166/catedra.v2i3.1803 


\section{Procesos de innovación y cambio: visión panorámica}

Tal como lo han planteado Larrea y Granados (2016) en el caso de la educación superior ecuatoriana, surge el cuestionamiento en los actores y directivos del cambio educativo, con relación a si los modelos que se vienen aplicando desde hace décadas, pueden ser considerados como innovaciones o son modas eurocéntricas o novedades educativas del momento, por ejemplo, la orientación del currículo por competencias. De ahí la importancia de conocer el modelo y realizar una reflexión sobre las implicaciones de estas innovaciones en el quehacer educativo.

Ahora bien, el cambio es inherente a la evolución de la sociedad. En tal sentido:

La evolución de la humanidad es inherente a la noción de cambio en cada aspecto que la caracteriza: la producción de bienes de subsistencia, los modelos, los métodos para su explotación, la distribución y consumo de bienes, el proceso salud-enfermedad y su atención, la socialización, la comunicación, el arte y el ocio, entre otros. (García-Quintanilla et al, 2015, pp. 47-48).

Desde los inicios de la existencia de los seres humanos se han producido continuos procesos de cambio, que permitieron el crecimiento y desarrollo en todos los aspectos de la sociedad, en lo productivo, político, personal, la comunicación, el arte, entre otros elementos en pro de mejores condiciones de vida personal, familiar y comunal (García et al., 2015, pp. 47-53), sostienen que el cambio social es una construcción teórica con dos tipos de procesos:

- Los que mantienen la estructura y

- Los que tienden a cambiar.

Los dos procesos pueden presentarse en diferentes planos al mismo tiempo. Como ejemplo señalan a los padres de familia que pueden enseñar a sus hijos las costumbres con las que ellos fueron formados, pero a su vez pueden enseñarles pautas de conducta que les permitan adaptarse de mejor manera a la nueva estructura social. Se considera que el cambio no es una moda sino una estructuración teórica que organizan los pensadores sociales en cada época (García et al., 2015 p. 48).

En este sentido, en Ecuador, tal como señala Breilh, (2017), la Educación Superior vive un proceso de cambio para adecuarse a una nueva realidad, tiene un rol estratégico que cumplir, estableciendo procesos de desarrollo sustentable, dejando atrás modelos tradicionales, creando corrientes universitarias más avanzadas acorde con los diferentes momentos que caracterizan un cambio de época. Es responsabilidad de las instituciones universitarias potenciar el desarrollo de nuevas corrientes de pensamiento que den paso a un nuevo ethos universitario.

Es indudable que la sociedad a nivel mundial y especialmente la ecuatoriana demanda cambios emergentes en la educación superior; ha sido y es una necesidad cumplir con lo más sustancial de la Declaración de Bolonia para la educación superior (1999) donde se hace énfasis en las reformas curriculares, la homologación de títulos, el aprendizaje permanente, el acceso a oportunidades de estudio, y la formación y promoción de la movilidad. Este proceso permite los obstáculos para el ejercicio efectivo de libre intercambio, nuevas metodologías y financiación, con el fin de que la academia genere procesos de cambio que estén acordes con una sociedad dinámica en constante transformación. 
Parte de estos procesos de cambio pasan por el análisis de la rectoría de los organismos que dirigen la Educación Superior y con firmeza emprender un proceso de recuperación de la autonomía universitaria, entendida esta como la particular relación dialéctica que se establece entre el estado y la universidad (Tünnermann, 2008, pp. 314-315), así como también la independencia de las universidades frente al Estado y al gobierno, su capacidad de autogobierno y administración.

Sobre el cambio educativo, Murrillo y Krichesky (2012) manifiestan: "Los cambios educativos son procesos que se desarrollan en una organización o institución educativa, en el que intervienen varios factores de manera simultánea y secuencial, implica fases o etapas planificadas a desarrollarse de forma continua y permanente", (p. 28). En efecto, los cambios en las instituciones cuando implican aspectos que afectan la cultura no ocurren en un solo momento. Su complejidad supone diversas etapas que van desde el reconocimiento hasta la aceptación y adaptación. Todo proceso de transformación supone un desafío tanto en la implementación como en la evaluación y no siempre se obtienen los resultados esperados, pues depende de varios factores, entre ellos de la dificultad tanto en aprender como en aplicar los constructos y colateralmente comprender, facilitar y medir las dimensiones de los procesos de cambio (Hall, 2014, p. 99). Factores como el desconocimiento, la comprensión de la innovación, la participación en la implementación son determinantes para la adopción de los procesos de cambio en las organizaciones.

Al investigar sobre el cambio educativo destacan los trabajos de Michael Fullan (2002a) quien desde los años 60 desarrolló investigaciones relacionadas con el estudio y la práctica de la innovación educativa en su tierra natal, Canadá. El denominó a esa primera época, "la era de la adopción" (1972-1982) porque consideró que los cambios iniciados en el contexto educativo tenían como propósito importar innovaciones para conseguir mejoras educativas. La intención era mejorar los resultados educativos, especialmente en los E.E.U.U., pues se consideraba que los mismos eran bajos con relación a los logros de otros países, en particular, la Unión Soviética. El objeto central de atención durante este periodo era la innovación y no los profesores. Existía poca conciencia de que las innovaciones creaban incertidumbre acerca de los roles que debían asumir los profesores, y que ello supondría ciertos desaprendizajes y reaprendizajes. En esta época según Fullan (2002a), "se ha producido muy poco cambio en la educación y los protagonistas tienen poca incidencia en este proceso siendo considerados como 'adoptantes pasivos'” (p. 6), es decir, en esta etapa el modelo se refiere a los docentes como los usuarios.

La siguiente década (1982-1992) la denomina "la década del significado". En esta etapa el cambio es observado en su conjunto desde todas las aristas, especialmente por parte de quienes lo van a aplicar. Otra característica de esta etapa es la visión de los cambios como parte de un proceso que comprende las fases de iniciación, implementación, continuación y resultado. Fullan (2002a), considera que "Aunque todavía era un proceso lineal, había por lo menos una flecha de dos sentidos entre cada fase". (p. 7). Ya no se trata de la mera adopción de una innovación sino de un proceso complejo de transformación en el campo educativo. En este caso el docente interactúa con los demás elementos del cambio en cada una de las fases.

En esta década se pone énfasis en el significado de la innovación y en la preparación del profesorado para obtener los resultados esperados. Fullan al respecto señala (2002a), "Si el cambio pretende tener éxito, los individuos y los grupos deben encontrar el significado tanto de lo que quiere cambiar como el modo de hacerlo...", (p. 7). 
A partir de 1992 Fullan nos habla de la "década de la capacidad del cambio". En esta década Fullan escribe la primera serie sobre el proceso del cambio, en la que pone de relieve el cuestionamiento, ¿Hay algo por lo que merezca la pena luchar? Básicamente integra orientaciones y recomendaciones a los docentes que enfrentan dificultades en la implementación de los procesos de cambio.

A partir del año 2002, Fullan escribe una trilogía para documentar los procesos de innovación educativa. El primer libro denominado, Las fuerzas del cambio: Explorando las profundidades de la reforma educativa (2002 a), donde enfatiza que una persona al involucrarse en las profundidades del cambio, también se convierte en parte de él. En el mismo señala que "Aprendemos que no es posible resolver el problema del cambio, pero que podemos aprender a convivir con él de una forma más preventiva y productiva" (p. 7). El segundo libro de la serie se denomina, Las fuerzas del cambio: La continuación, (2004), está dirigido al estudio de la dinámica del proceso del cambio basado en un importante componente empírico que le permite evidenciar la evolución de la reforma a nivel interno y externo y proponer nuevas perspectivas de mejoramiento. Fullan (2004) al respecto señala: "Vamos a analizar en profundidad el papel del conocimiento en el seno de las organizaciones que aprenden, así como el conocimiento y las conexiones externas" (p. 9). El tercer libro se titula Las fuerzas del cambio con creces (2007) donde se pone de relieve la continuación de los estudios sobre el proceso del cambio y se plantea una nueva propuesta que intentar cambiar el contexto en lugar de aceptarlo como algo conocido. Adicionalmente, pone de relieve la importancia de la sostenibilidad de las reformas, estableciendo la necesidad de considerar las mejores condiciones para que las mismas perduren.

En general, el nivel de cambios en los procesos educativos y en los mercados laborales tal como señala Rama (2015), "...actúa como un terremoto sobre la forma de funcionar los sistemas educativos, creando cambios necesarios y por ende oportunidades de inversión, de innovación y de desarrollo de nuevos modelos de gestión" (p.23).

Tal como se refiere en la tesis doctoral "Preocupaciones del profesorado ante la implementación de los rediseños curriculares en las carreras de educación de la Universidad Central del Ecuador" (Paez, 2018), el tercer milenio indudablemente significa enfrentarse a un cambio de época; y en estos nuevos contextos la educación debe concebirse de diferente manera y por ello en todos sus niveles se deben realizar investigaciones que permitan diagnosticar los nudos críticos y promover cambios. Los cambios se pueden realizar a través de reformas que generen transformaciones en la organización del conocimiento, del proceso de aprendizaje y la gestión académica, considerando siempre los nuevos horizontes epistemológicos, los nuevos escenarios sociales, políticos y culturales. $\mathrm{Si}$ se quiere ser actor de procesos de evolución, se debe responder a nuevos retos de cambio y transformación.

Tal como señala Fullan (2002b), el cambio es obligatorio en educación, los docentes deben tener una formación diferente al pasado; las instituciones educativas y el sistema mismo deben cambiar en cuanto a la formación de los futuros docentes; se deben establecer metodologías que relacionen al entorno y a la comunidad; se debe promover la reculturización, es decir, transformar los hábitos, habilidades y prácticas de los educadores y promover la creación de nuevas acciones. El cambio no debe ser impuesto; estos procesos no deben manejarse aisladamente. Se resalta la necesidad de establecer conexiones, redes, para enriquecer las propuestas, conocer las fortalezas y debilidades de este e ir solucionando en forma conjunta los problemas que surjan. 
Según Beraza y Zabalza, (2012), "Innovar es introducir cambios justificados" (p. 28), refiriéndose al hecho de que los cambios superficiales no benefician el proceso de inter aprendizaje. Por ello, deben considerarse cambios profundos para que impacten y beneficien a la comunidad educativa y a la sociedad. Estos cambios deben responder a las necesidades y demandas específicas del entorno.

Al mismo tiempo, tal como nos muestran Osorio y Pech (2007) no debemos considerar los procesos de cambio desde una sola dirección. "Los cambios no deben ser abordados desde una sola perspectiva, ya sea como política gubernamental o como surgida de la iniciativa de la escuela, sino que desde ambas direcciones y en conjunto con las fuerzas sociales, tecnológicas, económicas, etc., circundantes" (p. 175). Ello nos ofrece, por una parte, una visión bastante compleja de lo que supone realmente los procesos de cambio y los factores que intervienen su desarrollo.

Por otra parte, es importante considerar que todo proceso de cambio, innovación o reforma educativa curricular debe ser conocido, construido e implementado por los actores educativos, es decir, por el profesorado, considerado como agente del cambio. Lamentablemente los actores del proceso reciben la información cuando tienen que aplicar los cambios, generándose una serie de preocupaciones, incertidumbres, malestar y resistencia, lo que incide en su implementación. Tal como han señalado Rutherford y Hall (1990), los cambios se favorecen en la medida en que intentan dar respuesta a una carencia o necesidad institucional; se presentan ante la institución y actores fundamentales de manera clara se expresan en su complejidad y viabilidad y se explican en cuanto a su alcance y diversidad. Una reforma o innovación parcial o total va a funcionar si existe por parte de los docentes la formación, convicción, de que la nueva propuesta curricular guarda correspondencia con los conceptos más amplios de innovación, es decir, que estén bien fundamentados, organizados, contextualizados, que sean viables y prácticos

En tal sentido, Havelock y Huberman (1980, p. 49). señalan que:

La fragilidad del proceso de innovación considerado como un sistema hace indispensable un profundo conocimiento de las características de su funcionamiento en relación con el contexto en que se desarrolla. Un proyecto innovador puede fracasar por razones muy diversas y solo una planificación cuidadosa e idónea podrá dar al innovador cierta seguridad de que estará en condiciones de coordinar el sistema y mantenerlo en funcionamiento (Havelock and Huberman, 1980, p. 49).

En consecuencia, si las reformas son impuestas desde las autoridades superiores externas o internas de las IES (Instituciones de Educación Superior), se creará preocupación e inclusive resistencia. Es imprescindible entonces que se realicen acciones concernientes a la socialización, concientización y aceptación por parte de los docentes, junto a la preparación conceptual y práctica sobre las innovaciones educativas. Los docentes han de contar con una capacitación que les permita alcanzar niveles de convicción de que las innovaciones las entienden, las pueden aplicar y comprenden su alcance.

Para la UNESCO innovación educativa es un acto deliberado y planificado de solución de problemas que apunta a lograr mayor calidad en los aprendizajes de los estudiantes, superando el paradigma tradicional. Implica trascender el conocimiento academicista y pasar del aprendizaje pasivo del estudiante a una concepción donde el aprendizaje es interacción y se construye entre todos (UNESCO, 2016, p.3). 
La innovación es la forma más idónea como las instituciones educativas responden a los requerimientos de una sociedad, a su complejidad actual. De acuerdo con Villavicencio (2017) existe actualmente el requerimiento de las instituciones de educación superior, especialmente de las carreras de educación en las que se forman los futuros docentes de todos los niveles educativos del Ecuador, de introducir modalidades alternativas de diseños curriculares en los que se consideren las actuales demandas sociales, la armonización de los nuevos horizontes epistemológicos, el creciente y acelerado cambio que se opera en ciencia y tecnología, con el fin de responder a los nuevos desafíos que debe enfrentar el ser ante la rapidez y profundidad de los cambios que se operan en la vida misma, en las familias, en las instituciones, en la sociedad. Según Pérez (2012) "La confluencia de cambios tan significativos y radicales está conformando un nuevo meta contexto que cambia las instituciones, los Estados y la vida cotidiana de los ciudadanos dentro de una era de globalización e interdependencia" (p. 49). El nuevo meta contexto se refiere a las nuevas relaciones políticas y económicas del Estado-nación, a los procesos de globalización en lo ambiental, cultural, político y a la interdependencia relacionada a la convivencia de nuevos grupos humanos diferentes y hasta discrepantes en ambientes que determinan la complejidad del contexto.

En estos procesos de cambio se debe involucrar a la sociedad, a las instituciones de educación tanto públicas como privadas y al profesorado que debe participar de manera decisiva y directa. Las instituciones educativas deben centrar su atención en el agente principal de los procesos de innovación, debe conocer sus pensamientos, expectativas, intereses personales y profesionales, la formación recibida, aptitudes y preferencias.

En las investigaciones de Beraza y Zabalza (2012), dedicadas a la dimensión institucional y organizativa en las innovaciones educativas, los autores destacan el rol fundamental de los profesores innovadores en la implementación de la innovación. Se destaca el papel determinante de la cultura institucional en lo concerniente a la percepción que tienen los docentes que forman parte de la institución, percepción que se manifiesta en el significado y valor que los docentes le atribuyen a la innovación. El proceso de cambio es difícil de comprender y aceptar, más aún si la persona, organismo o institución no ha sido invitado a participar en su construcción.

Tal como afirma Fullan (2002b), las reformas educativas corren el riesgo de fracasar en la implementación si no se consideran los elementos básicos como la evaluación de los anteriores procesos de cambio, determinando sus fortalezas y debilidades; además, si no se toma en cuenta a los actores del cambio o si intervienen factores de otra índole como políticos, religiosos que quieren imponer tendencias que van dirigidas al cumplimiento de otros intereses, distantes de la calidad de la educación y la formación integral de los futuros profesionales.

Cuando se habla de cambios se suelen utilizar indistintamente los términos cambio, reforma o innovación. Se hace entonces necesario precisar el significado de estos términos. Casanova (2015), resume el significado de los tres términos de la siguiente manera: "el cambio como el hecho de dejar de hacer una cosa para hacer otra, la reforma como volver a hacer, modificar las situaciones para corregirlas y mejorarlas y la innovación como la alteración de algo para introducir novedades" (p. 17) siendo la innovación "La incorporación de algo nuevo dentro de una realidad existente en cuya virtud, esta resulta modificada” (p.20).

Por su parte, Fullan, (2002b) señala que el cambio es multidimensional y supone al menos tres componentes: utilización de materiales didácticos, nuevos enfoques didácticos y nuevos enfoques teóricos. Y manifiesta que “... el problema de mucha de la bibliografía 
hasta el momento es que el objeto central hace énfasis en la innovación y no en el usuario (padres, profesores y alumnos)" (p. 4). En tal sentido, hace énfasis en el estudio del artefacto o la metodología que es el objeto que debe provocar el cambio y no en los efectos que dicho objeto provoca en los usuarios de la misma. Indudablemente, frente a todos los cambios que ocurren actualmente en el mundo, la educación debe responder con inclusiones permanentes y continuos procesos de cambio que requieren voluntad, compromiso, entusiasmo del profesorado, para que se produzca una efectiva participación durante el proceso que va desde la construcción de las innovaciones, su ejecución y la evaluación.

Murrillo y Krichesky (2012), sostienen que "posiblemente uno de los peores pecados de la educación es la autocomplacencia: quien crea que ya lo hace bien, que no necesita mejorar, está en el camino para hacerlo mal y cada vez peor" (p. 27). En todo proceso de cambio social, podemos señalar que, si no se avanza, se retrocede.

Para realizar cualquier proceso de cambio especialmente en educación se debe considerar su complejidad, conocer las etapas que lo componen y saber anticiparse a las resistencias propias que se originan en los docentes ante estos procesos. Concretamente se deben identificar las preocupaciones del profesorado con el fin de establecer las acciones necesarias que permitan superar los problemas personales, profesionales e institucionales e iniciar el proceso de cambio, de innovaciones, con mejores garantías de sostenibilidad y eficacia. En este sentido son significativos los estudios realizados por Hall, G. y Hord, S. (1987) donde se establecen las etapas de preocupación del profesorado ante las innovaciones.

Es necesario, tal como señala Casanova (2015) establecer procesos de cambio, innovadores y emancipadores para la construcción de una política pública y el desarrollo de reformas institucionales para la educación superior, que permitan establecer nuevas escalas y protocolos de evaluación, de aseguramiento de la calidad, (no de acreditación). Es también necesario establecer nuevos modelos educativos, propuestas curriculares, estrategias pedagógicas, para carreras de grado y programas de posgrado. A ello se suma la necesidad de dar respuesta a la creación de diferentes sistemas de admisión y nivelación, eliminando aquellos que profundizan las estructurales e históricas brechas sociales que dejan fuera de la educación superior a miles de bachilleres cada año, que pasan a formar parte de un grupo poblacional que no tiene oportunidad de forjar un futuro promisorio por la imposibilidad de lograr un cupo en las universidades públicas y de igual manera lograr un trabajo digno.

\section{Aspectos que intervienen en la innovación educativa}

La definición de innovación educativa no está clara, existen muchas definiciones. Por ejemplo, Rogers (2003) señala que "la innovación es una idea, práctica y objeto que es percibido como nuevo por un individuo o unidad de adopción” (p.20). En esta acepción, cualquier elemento que aparezca y altere la imagen que los individuos conserven de un entorno se considera como innovación. En el caso educativo podemos referir la incorporación de artefactos en el aula, la incorporación de una nueva práctica, una metodología, un nuevo instructor. Al mismo tiempo que puede considerarse como innovación la modificación del espacio físico, la incorporación de estímulos como colores o sonidos, etc. Como vemos la acepción es bien amplia y a partir de ella se pueden considerar muchos aspectos como innovación.

En el caso particular de la innovación educativa García-Quintanilla et al. (2015), manifiestan que una innovación educativa constituye un trabajo novedoso y de envergadura tendiente a completar o a crear un sistema o procesos de cambio intencional, constituido por un proyecto o un conjunto de actividades que son parte de un proyecto (p.50). A veces se considera que es sinónimo de cambio, pero no es así; se puede observar que están 
vinculados estrechamente entre sí, pues la innovación produce cambios, los mismos que se les considera especiales, pues constituyen un esfuerzo deliberado que tiene como fin principal el mejoramiento del sistema.

Beraza y Zabalza (2012) definen así la innovación: es un "esfuerzo deliberado con vistas a obtener mejoras importantes en el sistema" (p. 46). "Constituye un trabajo novedoso y de envergadura tendente a completar o a crear un sistema", (p.47). En una sociedad permanentemente se busca mejorar y potencializar resultados en todas las áreas y para ello se recurre a nuevos procesos, estrategias, actividades, que posibiliten alcanzar ese cambio anhelado. En el caso de las innovaciones educativas son procesos que surgen encaminados a introducir cambios fundamentales en una búsqueda incesante de soluciones a los problemas que van enfrentando las instituciones relacionados directamente con los cambios sociales, los nuevos paradigmas, los nuevos enfoques pedagógicos.

Como innovación educativa se identifica a una acción o acciones de cambio que de manera intencional se realiza en educación como parte de un proyecto. Tejada (1998) menciona que "toda innovación es siempre una acción deliberada y que dicha experiencia deberá tratarse con todo rigor" (p. 13). Cita como condicionantes para el cumplimiento de los objetivos en una institución la intencionalidad, sistematización, dimensión contextual, dimensión sustantiva, dimensión personal, dimensión procesual y dimensión evaluadora.

Al referirse a la innovación educativa plantea que es necesario que se revise el significado del término innovar, ya que, si bien es cierto que en educación existe un efervescente mundo de innovaciones, éstas deben ser aplicadas con mucha reflexión. Tiene que establecerse lo que significa una innovación y qué no lo es, "innovar no es sólo hacer cosas distintas, sino algo mejor que lo anterior" (Beraza y Zabalza, 2012, p.19).

Para poder desarrollar procesos de innovación educativa debe primero existir la decisión de innovar, luego la comprensión del proceso, el análisis de la información, el establecimiento de las prioridades, la visualización de la situación, la definición de las estrategias, la instrumentación del plan, la evaluación y la gestión del cambio (Ortega et al., 2007).

En la construcción de las innovaciones debe existir la participación directa de los actores educativos y sociales, maestros y estudiantes, toda la comunidad educativa. Como ocurre actualmente en el Ecuador, estos cambios son impuestos, al igual que las normativas que asfixian a las instituciones de educación superior, pues responden a un modelo tecnocrático, lineal, concentrador, basado en esquemas hegemónicos, euro centristas, funcionalistas, que han puesto a las IES y a los docentes en el sendero de una educación elitista, de una meritocracia individualista, creando espacios para un fenómeno nacional y mundial. Los Rankings, según Villavicencio, (2017), muchas veces tienen efectos perversos ya que pueden afectar a determinados grupos sociales, a las instituciones educativas, a desdibujar la visión de la gestión académica (investigación, docencia y vinculación) dando paso a las preocupaciones administrativas, (llenado de matrices y acumulación de evidencias).

Es imperativo el cambio, la innovación, las reformas en educación, pero estas deben ser construidas con el profesorado, con toda la comunidad educativa, y para ello se deben establecer estrategias que posibiliten su aplicación.

Al referirse al proceso de innovación Rogers (2003), presenta las siguientes etapas:

\begin{tabular}{|c|c|}
\hline Escenario & Definición \\
\hline Conocimiento & Se conoce y entiende la innovación \\
\hline Persuasión & $\begin{array}{l}\text { Se desarrolla una actitud a favor o en contra de la innovación y se } \\
\text { argumente }\end{array}$ \\
\hline
\end{tabular}

Revista Cátedra, 2(3), pp. 83-103, septiembre-diciembre 2019. e-ISSN: 2631-2875

https://doi.org/10.29166/catedra.v2i3.1803 


\begin{tabular}{ll}
\hline Escenario & Definición \\
\hline Decisión & Se acepta la innovación o se rechaza definitivamente y no se \\
& continúa con el proceso \\
Implementación & Se pone a prueba la implementación de la innovación \\
Confirmación & Se refuerza la aceptación de la innovación
\end{tabular}

Cuadro 1. Etapas del proceso de innovación. Fuente: (Rogers E. 2003. Diffusion of innovations)

Las etapas del proceso de innovación presentado por Rogers (2003) expresa claramente que un proceso de innovación, especialmente curricular, implica el manejo de información de manera amplia, clara, correcta y definida, información que permita al profesorado reducir los niveles de incertidumbre que ocasiona la desinformación o la información equivocada con relación a los cambios a adoptarse. El conocimiento potencial le permitirá entender la innovación y tener los argumentos necesarios para tomar una posición frente al cambio y decidir la adopción o no del mismo. Si es favorable la decisión se continuará con la implementación de esta que estará determinada por la complejidad de la estructura y de los recursos de que disponga la institución para su aplicación y con el convencimiento de su funcionalidad, oportunidad, pertinencia, aspectos necesarios para lograr una acción transformadora y de impacto en los múltiples ámbitos de la institución educativa y de la sociedad.

Indudablemente, tal como señala Ortega et al. (2007) a la hora de realizar cambios, es importante contar con docentes innovadores, preparados para desarrollar estrategias de cambio y reformas, basados en la investigación y la vinculación, dispuestos a realizar un trabajo colaborativo, inclusivo, que desarrollen su gestión en procesos de internacionalización y virtualización de los aprendizajes. Condiciones que son indispensables, esenciales si se considera a la innovación no como un cumplimiento formal burocrático de lo impuesto por la administración de organismos o autoridades superiores (p.153).

Los procesos de innovación en su implementación tienen factores adversos que muchas veces dificultan cumplir con los objetivos esperados. Havelock y Huberman (1980) se refieren a seis factores como adversos al proceso de innovación:

1. Subestimación del proceso de innovación: coordinación y comunicación insuficientes.

2. Conflictos y motivaciones personales: oposición al cambio, motivaciones personales, dificultad para comprender a los otros.

3. Subdesarrollo: condiciones negativas en recursos, insuficientes materiales.

4. Problemas financieros: insuficientes aportes financieros.

5. Oposición de grupos influyentes: ideas contrarias de grupos de poder, del grupo dirigente.

6. Malas relaciones sociales: falta de armonía entre los miembros, dificultad de relación, ideas contrarias (pp. 304-323).

En efecto, el éxito de la implementación de las innovaciones curriculares depende de que los factores adversos a la innovación ya reseñados, sean puestos en evidencia con el fin de superarlos en la institución educativa. Ello es posible en la medida en que los actores se 
familiarizan con la propuesta de transformación. Se debe pensar que debe existir una coordinación y comunicación suficiente con el profesorado antes de iniciar el proceso de cambio, con el fin de que conozcan, discutan su aplicación y se familiaricen con ella. También es necesario que se desarrolle un proceso de motivación para ir superando actitudes opuestas al cambio, conociendo las razones y analizando las mismas. Se debe contar con los recursos suficientes tanto humanos, materiales, como financieros para atender los requerimientos exigidos y realizar reuniones de trabajo que permitan encontrar acuerdos y armonía entre los actores vinculados al proceso.

Es importante destacar que no es suficiente la buena intención por parte de autoridades y profesores para alcanzar el éxito esperado con las innovaciones propuestas. Es necesario que los docentes asuman como propio el proyecto innovador y se comprometan con los procesos de cambio y de co-construcción que favorezcan y fortalezcan una educación de calidad, actualizada en los planos científicos, tecnológicos, curriculares, culturales y ciudadanos. Las instituciones educativas en Ecuador generalmente desarrollan procesos de cambio, introducen innovaciones, nuevos programas, prácticas, diseños curriculares, con la intención de generar mejores resultados para los estudiantes, para la comunidad, procesos que conllevan dificultades en su implementación y no siempre se consigue buenos resultados, inclusive en ocasiones los resultados son desfavorables. Cuando eso sucede a menudo prueban otras reformas o innovaciones, obviando conocer y comprender las causas que no permitieron cumplir con los objetivos esperados.

El siglo XXI exige que la educación se desarrolle con una nueva visión; deben transformarse los organismos rectores de la educación, las instituciones educativas, los modelos educativos y pedagógicos, los diseños curriculares, sus programas; especialmente debe cambiar la formación del docente con el fin de que su desempeño responda a los nuevos horizontes epistemológicos, a los nuevos retos determinados por la complejidad, la modernidad. Al respecto Fuguet (2015) dice, "Sin duda alguna, un reto educacional es poder afrontar los cambios continuos en los avances tecnológicos, especialmente los relacionados con los de la comunicación e información" (p. 100).

En lo referente al docente universitario, señala Mas-Torrelló y Olmos (2016) , que este debe considerarse un profesional en permanente proceso de cambio, debido a las transformaciones epistemológicas, paradigmáticas, refiriéndose por ejemplo a no centrar la atención en la enseñanza y en el profesor, sino a centrarse en el aprendizaje y el alumno, los cambios sociales, culturales y estructurales, los nuevos diseños curriculares, la revisión de las metodologías utilizadas, los nuevos modelos de evaluación, de rendición de cuentas, la aplicación de nuevas normativas refiriéndose a leyes, reglamentos, instructivos entre otros aspectos y a los acuerdos, resoluciones, mandatos de convenciones nacionales e internacionales que plantean recomendaciones sobre el requerimiento de profundos cambios por la calidad y la internacionalización que deben ser asumidas por las universidades (Mas-Torrelló and Olmos, 2016).

Esta realidad conduce a modificaciones en las funciones, roles y tareas asignadas al profesor y la exigencia de adoptar nuevas competencias para desarrollar eficientemente sus funciones profesionales, lo que a su vez origina la necesidad de establecer planes de educación continua encaminados a definir y orientar los nuevos roles en el cumplimiento y la articulación de las funciones sustantivas del docente en los diferentes escenarios que demanda la educación del siglo XXI. La innovación se constituye entonces como un camino que incide en algún aspecto estructural y funcional de la educación para mejorar su calidad.

Con relación a las preocupaciones de los docentes por las innovaciones curriculares, Hall y Hord, (citado en Marcelo, Mayor y Sánchez, 1995), manifiestan que "una preocupación sería la representación compuesta de sentimientos, inquietudes, pensamientos y consideraciones 
dadas a una cuestión particular o a una tarea" (p.154), en consideración a que cada profesor percibe las situaciones de forma diferente en relación con el hecho que enfrenta de acuerdo con su propio esquema de desarrollo.

Preocupación significa estado de inquietud y temor producido por una situación problemática. Si este estado se presenta en procesos de cambio, innovación, puede convertirse en obstáculo en el momento de aplicar las reformas o innovaciones y los resultados seguramente no serán los esperados, corriéndose el riesgo de fracasar, ya que éstas pueden ser adoptadas o rechazadas individual o colectivamente.

Las personas, al experimentar los cambios, tienen muchos cuestionamientos sobre cómo les afectará, cómo lo realizarán y qué impacto tendrá en los estudiantes, en la institución; tienen escepticismo e incredulidad con respecto a la utilidad y validez de los procesos y de quienes dirigen, inclusive desconfianza, interrogantes que al no ser contestadas, pueden generar resistencia, indiferencia, desmotivación y hasta oposición, todos ellos considerados factores determinantes al momento de implementar cualquier proceso de cambio.

Fullan (2004) sostiene que, para alcanzar éxito en la aplicación de un proceso de cambio educativo, se debe, entre otros factores, comprender y fusionar la fuerza intelectual, política y espiritual, las mismas que deben desarrollarse y combinarse. La fuerza intelectual, referida a la creación de conocimientos sobre el proceso del cambio; la fuerza política, que establece procesos de trabajo colaborativo, con alianzas internas y externas; y la fuerza espiritual o propósito moral que implica provocar el debate y el compromiso con el cambio. Concluyendo Fullan (2004) dice: "Es evidente, además, por qué necesitamos el poder de fusión, es decir, que las tres fuerzas interactúen y se combinen para conseguir el efecto máximo" (p. 97).

\section{Factores que deben intervenir en el cambio educativo}

El proceso de cambio educativo comienza con la identificación de un problema y en el caso de la educación superior en el Ecuador, los organismos que ejercen la rectoría de la misma realizaron evaluaciones en las que se identificaron varios problemas y serios cuestionamientos como consta en el informe del Consejo de Educación Superior (CES, 2014).

En este contexto, valga destacar que García-Quintanilla et al (2015), presentan tres principios básicos que deben orientar los procesos de cambio en educación:
1. Aumento en la participación democrática: la dirección de los procesos y actividades debe favorecer un ejercicio más libre de la opinión y el surgimiento de iniciativas a través de un proceso de plena comunicación. 2. Valoración crítica: encaminado a conocer y analizar qué se hace, para qué se hace y cómo se hace.
3. Motivación: en el proceso de cambio debe existir la oportunidad de una mayor implicación profesional del personal, con base en actividades motivacionales (p. 49).

Los cambios en educación constituyen un proceso complejo cuyas etapas deben ser conocidas por quienes los van a aplicar. Además, los líderes deben anticiparse a las posibles actitudes de resistencia que se generen y establecer las estrategias para iniciar, ejecutar y evaluar el proceso y avalar su eficacia y sostenibilidad (Murrillo y Krichesky, 2012,p. 27).

Fullan, M. y Levin, B. (2009) en una investigación realizada en Toronto, Canadá, establecen que el cambio en educación debe ser políticamente impulsado por los líderes quienes deben 
comprender, aceptar y participar mediante el establecimiento de un conjunto de estrategias fundamentales de todo el sistema de reforma. Los autores plantean seis fundamentos:

1. Desarrollo de la profesión docente: la premisa básica es el respeto a los docentes y al conocimiento profesional. No se puede aplicar la reforma en conjunto a menos que todos los docentes se encuentren trabajando para alcanzar un mismo objetivo. Además, se necesita una combinación de paz laboral, estabilidad e incentivos para que la profesión se desarrolle.

2. Establecimiento de objetivos medibles en colaboración con todos: es necesario establecer metas específicas en asociación con todos los involucrados, sean instituciones, o grupos de docentes.

3. Mantener una calle de dos vías entre la instrucción y la evaluación: la buena instrucción debe conducir a la evaluación y una evaluación debe conducir a una buena instrucción. Las dos deben permitir el desarrollo de las capacidades.

4. Reconocer al liderazgo como un elemento clave en el proceso de cambio: es preciso fomentar el liderazgo efectivo de todos los participantes con roles directivos.

5. Establecer estrategias de intervención integral: basados en la motivación a todo el personal inmerso en el proceso de cambio para lograr desarrollar la colaboración integral.

6. Asignar los recursos para todos los proyectos y utilizar los existentes: implica poner al servicio de la ejecución de los proyectos los recursos económicos requeridos, de una manera enfocada (pp. 30-31).

Sobre el cambio educativo en la universidad ecuatoriana, Ramírez (2016), dice "Este es quizás uno de los principales retos que tiene la universidad ecuatoriana; no solo trasmitir conocimiento sino tener un pensamiento crítico-reflexivo, generador de conocimiento autónomo, responsable frente a los intereses comunes que tenemos como sociedad, región y mundo", (p. 47). El cambio en educación superior permite el desarrollo de la sociedad y por ende el acrecentamiento de la calidad de vida de los seres humanos, pues es un medio cultural que posibilita descubrir y cultivar la individualidad y fortalecer la convivencia en colectividad en pro del cumplimiento de los grandes objetivos. Los objetivos se logran con base en las transformaciones de las universidades para responder a los constantes cambios que plantea el contexto mundial, regional y local actual.

En los cambios educativos intervienen muchos factores de índole personal, conductual y cultural que favorecen u obstaculizan los mismos. Según Sepúlveda y Murrillo (2012) estos factores son:

Factor personal: por las resistencias u obstáculos que impiden estar abiertos a los cambios que se presentan por las creencias que se forman a lo largo de la vida. Estos son los modelos mentales que condicionan determinadas formas de pensar y actuar hasta llegar inclusive a considerar aquello como única verdad.

Factor conductual: por creer que no se está preparado para los cambios, pues las nuevas ideas quitan la seguridad o tranquilidad y restan confianza para poder seguir con aprendizajes nuevos.

Factor cultural: ya que cada persona aprende e interioriza a lo largo de la vida, costumbres, normas y conductas que influyen en su vida (pp. 8-9). 
Es importante señalar que en el desarrollo de todo proceso de cambio educativo se deben considerar todos los factores, internos y externos a la institución y especialmente los docentes, ya que no hacerlo puede determinar que se actúe en dirección contraria, lo cual seguramente generará resistencias, o indiferencia y/o temores que pueden alterar el proceso o limitar el alcance de la innovación.

En síntesis, pareciera que la tendencia es a realizar un trabajo colectivo en cuanto se refiere a cambios de trascendencia que revolucionen la educación como repuesta a los innumerables problemas que afectan a las sociedades del mundo y de manera particular a Latinoamérica. Se debe abrir un camino para adelantar los cambios educativos, pedagógicos, curriculares, metodológicos, conscientes de que el cambio en educación es perentorio y se debe aplicar en pro de alcanzar una educación de calidad, contextualizada, moderna, para la diversidad, interculturalidad, equidad e inclusión, que sea sostenible y se pueda generalizar.

\section{Conclusiones}

Para desarrollar un proceso de cambio educativo, es necesario que la comunidad construya una propuesta en la que se integren y articulen los elementos pertinentes, desde la fundamentación filosófica, epistemológica, sociológica, psicológica, pedagógica; los principios y objetivos del proyecto; hasta las estrategias, en las que conste la sensibilización y capacitación a los docentes, entre otros elementos. Estos aspectos posibilitan que el profesorado se sienta involucrado y logre entusiasmarse al formar parte activa de un gran proyecto.

Se debe reconocer que un factor que a menudo se pasa por alto en los procesos de cambio es el elemento humano, pues no se considera que los docentes son los actores educativos, los profesionales que realmente hacen el trabajo; además, generalmente no se toma en cuenta que cada persona responde a un nuevo proceso con actitudes, creencias, con un historial personal y profesional propio, hecho que determina que cada uno actúe de manera diferente frente a los procesos de cambio. El cambio de mentalidad es fundamental en todos estos procesos, pues de no existir se corre el riesgo de encontrar actitudes defensivas, superficiales, de éxito efímero (Fullan, 2002b, p.15).

$\mathrm{Al}$ referirse al cambio educativo se debe entender que el mismo no afecta solo al sistema escolar, sino también a las personas involucradas quienes presentan diferentes percepciones, actitudes, sentimientos y preocupaciones, las mismas que deben ser consideradas a fin de lograr una gestión que facilite e impulse el proceso de cambio que según Hall (2014), se tarda de tres a cinco años para que ocurra. Al respecto, Osorio y Pech, (2007), señalan que "frente a las reformas educativas existe un amplio consenso de que estas no son sustentables si los actores involucrados no participan en su diseño y ejecución..." (p. 174)

Indudablemente es necesario abordar el proceso de cambio desde la perspectiva de los profesores, de las preocupaciones que se generen a partir de las reformas o innovaciones que se quieran implementar, si se considera que son los actores directos de la aplicación en el aula, en la institución y que es básico acompañar a los profesionales en este recorrido para apoyar y afianzar el proceso.

Finalmente se confirma que se deben conocer las preocupaciones, sentimientos, inquietudes de los profesores en referencia a la reforma a aplicarse con el fin de predecir y determinar acciones para la preparación, la capacitación, la orientación, la asistencia y la consecución de los recursos necesarios para la implementación (Marcelo, Mayor y Sánchez, 1995). 
Para investigar las preocupaciones de los docentes frente a los procesos de cambio existen instrumentos validados en otras investigaciones (Martínez, 1999, Páez, 2018), tal como el Concern Based Adoption Model, (CBAM) de Frances Fuller, que es un modelo básico de adopción centrado en los concernimientos o preocupaciones que tiene el profesorado de manera individual en la implementación de un proceso de cambio, de reforma, de innovación en el área educativa. 


\section{Bibliografía}

Breilth, J. (2017). La universidad que pensamos y sus desafíos: Crítica al modelo tecnoburocrático. Las reformas universitarias en el Ecuador (2009- 2016) Extravíos, ilusiones y realidades. Quito. Ed. Universidad Andina Simón Bolívar.

Beraza, M.A. y Zabalza M.A. (2012). Innovación y cambio en las instituciones educativas. Rosario, Homo Sapiens Ediciones.

Casanova, M. (2015). La supervisión, eje del cambio en los sistemas educativos. Revista Iberoamericana sobre calidad, eficacia y cambio en educación. volumen 13. número 4. pp. 17 http://www.rinace.net/reice/numeros/arts/vol13num4/art1.pdf

Declaración de Bolonia para la educación superior (1999). Disponible en: http://www.educacion.gob.es/boloniaensecundaBarrigaria/img/Declaracion Bolo nia

Consejo de Educación Superior. (CES 2014).

Díaz-Barriga, F. (2012). Reformas curriculares y cambio sistémico: Una articulación ausente pero necesaria para la innovación. Revista Iberoamericana de Educación Superior, 3 (7), 23-40.

Fullan, M. (2002a). Las fuerzas del cambio. Explorando las profundidades de la reforma educativa. Madrid - España Ed. Akal, S. A.

Fullan, M. (2002b). El significado del cambio educativo: un cuarto de siglo de aprendizaje. Revista Currículum y Formación del Profesorado.

Fullan, M. (2004). Las fuerzas del cambio: La continuación. Madrid - España Ed. Akal, S. A.

Fullan, M. (2007). Las fuerzas del cambio con creces. Madrid - España Ed. Akal, S. A.

Fullan, M. y Levin, B. (2009). The Fundamentals of Whole-System reform. Education Week 28 (35), 30-31

García-Quintanilla, M., Serna, R.V., Gutiérrez, A.E. y Ruiz M. (2015). La teoría del cambio y la innovación como fundamento para la gestión del conocimiento. México. Ciencia

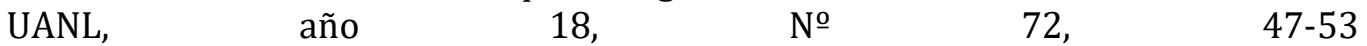
http://eprints.uanl.mx/6132/1/la\%20teoria\%20del\%20cambio.pdf

Hall, G. y Hord, S. (1987). Change in Schools. New York, State University of New York. Un instrumento para evaluar cambios en las etapas de preocupaciones de profesores. El inventario de preocupaciones de profesores. Sevilla. Universidad, Facultad de Educación. (p. 154).

Hall, Gene, E. (2014) Evaluando los procesos de cambio. Midiendo el grado de implementación. REICE, Volumen 12, № 4, especial. http://www.rinace.net/reice/numeros/vol12num4e.htm

Havelock, R.G. y Huberman, A.M. (1980). Innovación y problemas de la educación: teoría y realidad en los países en desarrollo. UNESCO. Colección: Estudios y encuestas de educación comparada.

Larrea, E., y Granados, V. (2016). El sistema de educación Superior para la sociedad del Buen Vivir basada en el conocimiento. El caso ecuatoriano. Guayaquil, Ecuador. Ed. Universidad Católica de Santiago de Guayaquil. pp. 160-169. 
Martínez, A. (1999). Computer Mediated Communication: Instructional Concerns in the College of Education and Health Professions in a land-grant university. A dissertation submitted in partial fulfillment of the requirements for the degree of Doctor of Education. University of Arkansas.

Mas-Torelló, O. y P. Olmos-Rueda. (2016). El profesor Universitario en el espacio Europeo de Educación superior: La autopercepción de las competencias docentes actuales y orientaciones para su formación pedagógica, Revista Mexicana de Investigación Educativa, 21(69). $\quad$ http://www.scielo.org.mx/scielo.php?pid=S1405$\underline{66662016000200437 y \text { script}=\text { sci arttext }}$

Murrillo, J. y Krichesky, G. (2012). El proceso del cambio escolar. Una guía para impulsar y sostener la mejora de las escuelas. REICE Revista Iberoamericana sobre calidad, eficacia y cambio en educación. Volumen 10 . número 1. http://www.rinace.net/reice/numeros/arts/vol10num1/art2.pdf

Ortega Cuenca, P., Ramírez Solís, M., Torres Guerrero, J., López Rayón, A., Servín Martínez, C., Suárez Téllez, L., Ruiz Hernández, B. (2007). Modelo de innovación educativa. Un marco para la formación y el desarrollo de una cultura de la innovación. RIED. Revista Iberoamericana de Educación a Distancia, 10 (1), 145-173.

Osorio, R. R. y Pech, C.S. (2007). Preocupaciones de los profesores ante la reforma integral de la educación secundaria en México. REICE. Volumen 5, № 3.

Páez, R. (2018). Preocupaciones del profesorado ante la implementación de los rediseños curriculares en las carreras de educación de la Universidad Central del Ecuador.Tesis Doctoral para optar al título de Doctora en Educación. Universidad Católica Andrés Bello. Caracas- Venezuela.

Pérez, G., A. (2012). Educarse en la era digital. Madrid. Ed. Morata.

Rama, C. (2015). La Universidad sin Fronteras. Editorial Universitaria. Lima-Perú. pp.23, pp. 74.

Ramírez, R. (coord.). (2016). Universidad urgente para una sociedad emancipadora. Quito. Ed. SENESCYT-IESALC.

Rogers, E. (2003). Diffusion of innovations. New York, Ed. The Free Press.

Rutherford, B. y Hall, G. (1990). Concerns of Teachers: Revisiting the original theory after twenty years. Paper presented at AERA.

Sepúlveda, C. y Murrillo, J. (2012). El origen de los procesos de mejora de la escuela. Un estudio cualitativo en 5 escuelas chilenas. Chile. REICE. Volumen 10, № 3./art1.pdf http://www.rinace.net/reice/numeros/arts/vol10num3

Tejada, J. (1998). Los agentes de la innovación en los centros educativos (profesores, directivos y asesores). España. DIALNET

Tünnermann Bernheim, C. (2008). La calidad de la educación superior y su acreditación: la experiencia centroamericana. Avaliação: Revista da Avaliação da Educação Superior (Campinas), 13(2), 313-336. https://dx.doi.org/10.1590/S141440772008000200005

UNESCO. (2016). Serie "Herramientas de apoyo para el trabajo docente". Texto 1 Innovación Educativa, 1aㅡ Ed. marzo. 
Villavicencio, A., (2017) Las reformas universitarias en Ecuador. Los rankings universitarios. Quito. UASB.

(c) (P) Licencia Creative Commons Atribución 4.0 Internacional (CC BY 4.0)

Revista Cátedra, 2(3), pp. 83-103, septiembre-diciembre 2019. e-ISSN: 2631-2875

https://doi.org/10.29166/catedra.v2i3.1803 


\section{Autores}

RUTH PÁEZ -GRANJA obtuvo su título de PhD. en la Universidad Católica Andrés Bello de Caracas Venezuela en 2018, es Magíster en Gerencia de la Educación Abierta en la Universidad Regional Autónoma de los Andes, UNIANDES del Ecuador, 2004. Obtuvo. Especialista en Docencia Universitaria, 2002. Diplomado en Inteligencia Emocional y Desarrollo del Pensamiento, 2001. Diplomado en Docencia Universitaria, 2006. Doctora en Psicología Educativa, 1982 y Licenciada en Psicología Educativa por la Facultad de Filosofía, Letras y Ciencias de la Educación de la Universidad Central del Ecuador en 1974.

Ex Decana de la Facultad de Filosofía, Letras y Ciencias de la Educación de la Universidad Central Ecuador, hasta abril 2018, actualmente profesora principal a tiempo completo de la cátedra de Proyectos de Investigación de la Carrera de Educación Inicial de la Facultad de Filosofía, Letras y Ciencias de la Educación de la Universidad.

ANA BEATRIZ-MARTÍNEZ obtuvo su doctorado en Educación por la Universidad de Arkansas. Profesora Titular de la Universidad Central de Venezuela. Ha realizado pasantías de investigación en la University of South Florida, USA; Universitá degli studi di Padova, Italia; Universidad de Montreal, Canadá y en Cornell University, USA. En la Universidad Central de Venezuela ha sido directora de la Coordinación Central de Extensión, Coordinadora General del Sistema de Actualización Docente del Profesorado y Coordinadora de la Comisión de Educación a Distancia.

Es profesora del Doctorado en Educación de la Universidad Central de Venezuela. Junto con otros investigadores ha sido compiladora y autora de Nuevos ambientes de enseñanza. Miradas iberoamericanas sobre tecnología educativa, Caracas, Edit. El Nacional. 2010; Teoría y práctica de las comunidades virtuales de aprendizaje, Caracas, Edit. CDCH-UCV. 2013; Diseño y Tutoría Virtual, Caracas, Edit. FHE-UCV. 2014; Comunicación y Aprendizaje en el ciberespacio. Las Comunidades Virtuales, Caracas, Edit. CDCH-UCV. 2010; Redes sociales comunicación y educación. Abediciones UCAB. 2017. Ha publicado diversos trabajos en revistas nacionales e internacionales. Entre sus reconocimientos destacan la beca Fulbright, el reconocimiento en el Sistema Nacional de Promoción al profesor investigador y la Orden José María Vargas de la Universidad Central de Venezuela. 